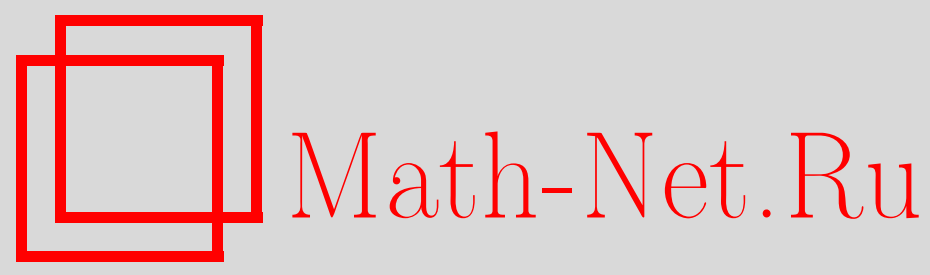

В. В. Шокуров, О рациональной связности, Матем. заметки, 2000, том 68, выпуск 5, 771-782

DOI: https://doi.org/10.4213/mzm997

Использование Общероссийского математического портала Math-Net.Ru подразумевает, что вы прочитали и согласны с пользовательским соглашением http://www.mathnet.ru/rus/agreement

Параметры загрузки:

IP : 54.92.164.108

26 апреля 2023 г., 16:16:25

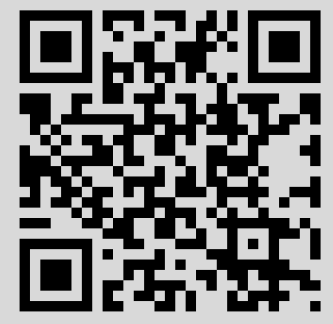




\title{
О РАЦИОНАЛЬНОЙ СВЯЗНОСТИ
}

\author{
В.В. Шокуров
}

Работа посвящена изложению доказательства одной из самых важных теорем бирациональной геометрии многообразий Фано.

Библиографоия: 17 названий.

Настоящая заметка является второй в серии "Letters of a bi-rationalist" (cм. [1]). Haписана она 5 октября 1995 г.; окончательная редакция - 12 марта 1997г. в Балтиморе. Она посвящается памяти одного из зачинателей современной бирациональной алгебраической геометрии Бориса Мойшезона.

Алгебраическое пространство $X$ рачионально связно, если любые его две точки в каждой компоненте связности $X$ можно соединить цепочкой рациональных кривых. Это равносильно $R$-эквивалентности всех точек каждой компоненты связности $X[2$, лемма 4.2]. Собственно говоря, это даже не связность, когда $X$ несвязно. $\mathrm{K}$ тому же в общем случае данная связанность слабее, введенной Кампаной в [3] и Колларом-МияокойМори (см. [4, определение-замечание 2.2]), о чем подробнее изложено в следствии 8 и замечаниях 2, 5. Однако, как ожидается, в случае (слабых) многообразий лог-Фано все они совпадают на каждой неприводимой компоненте связности (см. замечание 2).

Настояшая заметка имеет два источника. Первьй - рациональная связность неособых многообразий $Ф$ ано [5, следствие 3.2], используемая при доказательстве методом Фано ограниченности модулей последних [6, теорема 5.4 и следствие 5.6], [4, следствие 3.11]. Второй источник - вопрос А. Борисова о рациональной связности разрешения трехмерной лог-терминальной или даже терминальной особенности.

Мы придерживаемся в основном терминологии, обозначений и ограничений из [1], $[7]-[9]$.

Гипотеза о рациональной связности. Рассмотрим лог-пару $(X / S, B)$, состоящую из алгебраического пространства $X / S$ с гранищей $B$ и удовлетворяющую следующим условиям:

1) $X$ нормально (или, возможно, полунормально);

2) $f: X \rightarrow S$ собственно;

3) $K+B$ лог-каноничен;

4) $-(K+B)$ численно обилен $/ S$.

Работа выполнена при частичной поддержке фонда NSF, грант № DMS-9500971. 
Тогда все слои $f$ рационально связны.

Однако отдельные компоненты слоя могут быть не связны (см. замечание 2).

ПРИМЕР-ЗАмЕчАнИЕ. Рассмотрим неособую линейчатую поверхность $X / E$, где $E$ неособая кривая рода $g \geqslant 1$, являющаяся одновременно сечением $E \subset X / E$. При подходящем выборе $X$ и $E$ можно считать кривую $E$ исключительной с $E^{2} \leqslant-2 g+1$. Тогда имеется рациональное число $b$ такое, что $1 \leqslant b<2$ и лог-дивизор $K+D$ с $D=b E$ численно тривиален на $E$. С другой стороны, $-(K+D . C)=2-b>0$ на кривой $C$ слоя. Значит, $-(K+D)$ численно эфективен и, более того, объемен. Однако поверхность $X$ не рационально связна.

Тем самым в общем случае условие обильности 4) гипотезы нельзя ослабить до численной эффективности и объемности / $S$. Оданако последнее возможно при ослаблении особенностей лог-дивизора (см. следствия 4-6).

Сдувая $E$ в точку, мы получаем нормальную проективную поверхность дель Пеццо $Y$, предполагая $(K+D) \sim_{\mathbb{Q}} 0$ на $E$, что вьполнено для подходяшего нормального пучка $E$ на $X$. В этом случае $Y$ рационально связно, хотя и имеет не лог-каноническую особенность при $g \geqslant 2$. Заметим, что особенность в данном случае изолирована. Как будет видно из дальнейшего, условие 3) гипотезы на особенности можно ослабить в том же стиле до условия

$\left.3^{\prime}\right) K+D$ с эффективным дивизором $D$ (вместо границы $B$ ) лог-каноничен во всех подмногообразиях $Y \subset X / S \mathrm{c} \operatorname{dim} Y / S>0$.

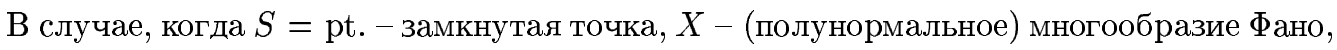
лог-каноничное вне конечного множества замкнутых точек (изолированных лог-особенностей) (ср. следствие 6).

Если мы чуть увеличим $b$ в предшествующем примере, то $X$ будет многообразием лог-Фано для $K+D$. Однако оно будет иметь лог-особенность $D$ дивизориального типа: условие $3^{\prime}$ ) гипотезы не выполнено. Значит, дальнейшее размазывание особенностей невозможно.

В трехмерном случае можно построить подобные примеры с $D=B=0$.

Пример 1. Пусть теперь $X / E$ - гладкое расслоение на коники, а $E=E_{1} \times E_{2}-$ произведение двух неособых кривых рода $g_{1} \geqslant 2$ и $g_{2} \geqslant 1$ соответственно. Снова при подходящем выборе $X$ и $E$ имеется рациональное $b$ такое, что $1 \leqslant b<2$ и лог-дивизор $K+D$ с $D=b E$ численно тривиален на $\left(E_{1}, \mathrm{pt}.\right)$, но отрицателен на (pt., $\left.E_{2}\right)$. Сдувая $E$ послойно над $E_{2}$ в кривую $E_{2}$, мы получаем трехмерное многообразие вою $E_{2}$ лог-особенностей. Кроме того, $Y / E_{2}$ - расслоение на дель Пещо с сечением $E_{2}$. Оно не рационально связно.

В предыдущем примере можно изменить $E$ так, чтобы $K+D$ было численно тривиально на $\left(\mathrm{pt} ., E_{2}\right)$. Тогда $Y$ - многообразие $Ф$ ано в слабом смысле: $-K_{Y}$ численно эффективен и объемен. Чтобы особенности на $Y$ (вдоль $E_{2}$ ) были лог-каноническими, надо взять конструкцию с $g_{1}=g_{2}=1$. При этом несвязность сохраниться.

В заключение отметим, что $X$ станет связным, если $E$ в предшествуюших примерах сдуть в точку. Это, в частности, показьвает, что рациональная связность не является бирациональным инвариантом в общем случае (тем не менее, ср. следствие 8). 
ТЕорема 1 (ср. [1, теорема 2]). Гипотеза о раииональной связности верна в размерности $\leqslant n$, если

1) для каждой лог-пары $(X, B) c \operatorname{dim} X \leqslant n$ сущ,ествует проективное лог-разрешение $Y \rightarrow X / S$

2) стандартные гипотезы о лог-моделях (СГЛМ) [9, 5.1] выполнены в размерностu $\leqslant n$.

В характеристике 0 по Хиронаке достаточно условия 2).

Теорема по существу вытекает из следующего ослабления.

ЛЕмма 1 (ср. [1, лемма 3]). В предположениях 1), 2) теоремы 1 пусть $X / S$, как в гипотезе о рациональной связности, а подпространство $E$ состоит из компонент вырождения

$$
\operatorname{Exc}(f / P):=\{x \in X \mid f(x) \in P \text { иморфизм } f \text { не конечен в } x\}
$$

над замкнутым подпространством $P \subseteq S$. Пусть $\gamma: E \rightarrow G / S$ рачионально собственный и доминантный морфизм алгебраических пространств. Тогда на $E$ имеется семейство $\left\{C_{\lambda}\right\} / S$ (возмохнн, несвязное) рачиональных кривых, покрывающее $G$; точнее $\left\{\gamma\left(C_{\lambda}\right)\right\}$ покрывает $G$.

Результат верен при замене условия 3) гипотезы на $\left.3^{\prime}\right)$.

В характеристике 0 достаточно условия 2).

ЗАмЕчаниЕ 1. Как будет видно из методов доказательства, лемма 1 справедлива также для некоторого подпространства $E^{\prime} \subseteq Y$, доминирующего $E$ на произвольном раздутии $X$. Действительно, по предположению 1) теоремы 1 раздутие можно считать достаточно произвольным, но фиксированным затем разрешением особенностей, например, лог-разрешением. При этом $E$, предполагаемое неприводимым, заменяется на доминируюший дивизор $E^{\prime}$ в случае $E \neq X$ и на разрешение $Y$ в противном случае, т.е. на многообразие $Ф$ ано $/ P=S$. Индукция по размерности сохраняет утверждение и приводит к требуемому доминирующему дивизору в случае не многообразия $\Phi$ ано $/ P=S$.

Согласно теореме Тзена утверждение не зависит от разрешения.

ДоКАЗАТЕЛЬСТВо ЛЕМмы 1. Можно восполься доказательством [1, лемма 3$]$ со следующими изменениями. Поскольку нас интересует общий слой $\gamma$ при $G \neq \mathrm{pt}$., мы можем пренебречь особенностями, не доминирующими $G$; поэтому - ограничиться условием 3) гипотезы.

Также предполагаем, что $E$ неприводимо и $f(E)=$ pt. Отметим, что рациональная цепочка деформируется в такую же цепочку.

Однако, $G \neq$ pt. предполагается всюду.

Затем согласно конструкции [1, лемма 3$]$, [8, эвристическое рассуждение] мы сводим задачу к случаю многообразия $\Phi$ ано: $S=\mathrm{pt}$. Для этого увеличиваем особенности $K+B$ в окрестности общего слоя $F$ проекции $\gamma$ так, чтобы они доминировали $G$ (точнее, би-дивизор с 0-й лог-дискрипантой), но в то же время были лог-каноническими в общем слое $/ G$. Например, если особенности совпали с $E$, но последнее условие не вьполнено, то доминируют не лог-канонические особенности и мы их понижаем до лог-канонических. 
Далее неприводимая и доминируюшая $G$ компонента особенностей и обозначается через $E$. Поскольку в окрестности полного подпространства (и даже проективного) $E$ имеется лиш конечное число центров с лог-дискрипантой 0 и $G \neq$ pt., такие центры доминируют $G$ вблизи общего слоя $F$.

Значит, лог-каноничность $K+B$ вблизи $F$ сохранится при добавлении $\varepsilon H$, где $H-$ общая гиперплоскость через дивизориальное семейство $F_{g}$, задающее дивизор Вейля $D=\cup F_{g}$ вблизи $F$. Заметим, что $D \neq 0$, так как $G \neq$ pt.

Более того, при малом $\varepsilon>0$ лог-дивизор $K+B+\varepsilon H$ по-прежнему отрицателен на $E$. Значит, при редукции случая многообразия $\Phi$ ано можно предполагать, что $K+B$ численно тривиален на $X=E$ и имеет $D \neq 0$ в $\operatorname{Supp}(B)$ послойного типа в окрестности общего слоя $F$. Теорема о свободе, как в доказательстве [1, лемма 3$]$, тут не нужна.

Вычитание $D$ сохранит численную тривиальность в общем слое $F$, но приведет к отрицательности на общих кривых поперек $F$.

Далее действуем, как в [1, лемма 3$]$.

ДоКАЗАТЕЛЬСТво тЕОРЕМЫ 1. Достаточно проверить требуемую связность для всех неприводимых компонент $E$ каждого слоя $f$. Последнее вытекает из [5, предложение 2.7], ибо условие 2) в данном предложении выполнено по лемме 1.

Согласно Риману лемма 1 верна и эквивалентна для нормализации $E^{\nu}$. Гладкость $E^{\nu}$ не требуется в доказательстве [5, предложение 2.7]. Однако проективность и нормальность, по-видимому, существенны.

СЛЕДСТВИЕ 1. Пусть $(X, D)$ - многообразие лог-Фано, лог-каноническое в размерности $\geqslant 1$, т.е. дивизор $-(K+D)$ обилен $и K+D$ имеет лог-канонические особенности вне конечного множества замкнутых точек. Тогда $X$ рачионально связно при выполнении предположений 1), 2) теоремы 1.

В характеристике 0 достаточно условия 2) теоремы 1.

Поскольку СГЛМ вьполнены в характеристике 0 и размерности $\leqslant 3$ [9], мы получаем

СлЕдСтвиЕ 2 (ср. следствие 4 ниже). В характеристике 0 гипотеза о рациональной связности и рациональной связности многообразия лог-Фано из следствия 1 верна при $\operatorname{dim} X \leqslant 3$.

СлЕДСТВИЕ 3. Предположим, что $g: Y \rightarrow X-$ собственный бирациональный морфизм (например, разрешение особенностей $X$ ), а $K+B$ лог-терминален. Тогда любой слой $g$ связен и рационально связен при выполнении предположений 1), 2) теоремы 1.

В характеристике 0 достаточно условия 2) теоремы 1.

ДокАЗАТЕЛЬСТво. Фиксируем точку $x \in X$ и докажем связность $g^{-1} x$. Утверждение локально по $X$.

Его достаточно проверить при $\mathbb{Q}$-факториальности $X$. Для чего заменяем $X$ на его $\mathbb{Q}$-факториализцию $X^{\prime} / X$, которая к тому же строго лог-терминальна. (Конечно, терминальность предполагает наличие подобного разрешения.) Тогда достаточно установить рациональную связность слоя $E \subset X^{\prime} / x$. Более того, нам надо найти рациональные кратные кривые сечения на некотором дивизоре $/ E$, доминируюшие $E$, указанного или доминирующего разрешения (ср. вопросы 1,2$)$. Последнее вытекает из замечания 1 
для $X^{\prime} / X$ и $K_{X}^{\prime}+B^{\prime}-\varepsilon H$, где $B^{\prime}$ - рациональньй образ $B$, а $H$ - относительное гиперплоское сечение. Отметим, что локально над $X$ можно считать $-H$ эффективньп и не содержашим центров с дискрипантами 0 . Значит, $K_{X}^{\prime}+B^{\prime}-\varepsilon H$ удовлетворяет условиям 1)-4) гипотезы при малых $\varepsilon>0$.

Заметим, что доминирующие рациональные цепочки могут оказаться несвязными. Однако они дополняются до связных кривыми слоя.

В $\mathbb{Q}$-факториальном случае, вычитая $B$, мы сводим задачу к чисто лог-терминальному случаю (с $B=0)$. Более того, тогда $X / X$ - единственная слабо лог-каноническая модель [7, 1.5.7]. Граница при этом считается приведенной на исключительных дивизорах пространства $Y / X$.

Достаточно установить связность для строго лог-терминальной модели $Y / X$, доминируюшей исходную (например, таком лог-разрешении особенностей $X$ ). Такая модель существует по предположению 1) теоремы 1 или по Хиронаке в характеристике 0.

Согласно предположению 2) теоремы 1 имеется последовательность (элементарных) перестроек $Y=Y_{N} \rightarrow Y_{N-1} \rightarrow \cdots-\rightarrow Y_{1}=X / X$. Поэтому можно воспользоваться индукцией по $N$.

Итак, предположим, что слой $Y_{N-1} / x$ рационально связен. Надо проверить, что то же самое верно для слоя $Y_{N} / x$. Согласно определению перестроек имеется диаграмма сдутий $/ X$ :

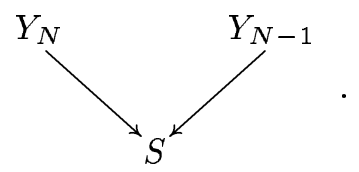

Тогда слой $S / x$ рационально связен как образ рационально связного. По теореме 1 и определению престройки все слои $Y_{N} / S$ рационально связны. Значит, слой $Y_{N} / x$ тоже рационально связен. Заметим для этого, что по $\mathbb{Q}$-факториальности $X$ исключительное множество $E_{N} / X$ дивизориально при его нетривиальности. Снова согласно замечанию 1 рациональные кривые, задающие связность, поднимаются как кратные сечения на любое разрешение или даже раздутие каждой компоненты пространства $E_{N}$. Последнее надо проверить для связности в слоях $Y_{N} / S$ покомпонентно. Это вытекает из того, что $K_{Y_{N}}+E_{N}$ лог-терминален и индуцирует стягивание теоремы 1 на каждой компоненте в $E_{N}$.

ТЕорема 2. Если в условиях теоремы $1 K+B$ Кавамата лог-терминален, то мы можсм усилить условие 4) гипотезы до следующих условий:

1) $-(K+B)$ численно әффективен и обтемен $/ S$;

2) выполнена теорема о свободе $[1,2.2]$.

В характеристике 0 достаточно условия 2) теоремы 1.

ДОКАЗАТЕЛЬСТВО-ЗАМЕЧАНИЕ. Как и для теоремы 1 , с заменой леммы 1 на $[1$, леммa 3].

Отметим также, что согласно условию 2) или [10, замечание 3-1-2] в характеристике 0 имеется сдутие $X \rightarrow Y / S$. Слои $Y / S$ рационально связны по теореме 1 , а слои $X / Y$ - по следствию 3 . Это влечет требуемое с точностью до существования кратных кривых-сечений (ср. вопросы 1, 2). Последнее выполнено по замечанию 1. 
СлЕДСТвИЕ 4 (ср. [4, следствие 3.11]). Пусть $(X, D)$ - слабое многообразие логФано, лог-терминальное по Кавамате, т.е. дивизор $-(K+D)$ численно әффективен, обвемен и $K+D$ лог-терминален по Кавамате. Тогда пространство $X$ рационально связно при выполнении предположений 1), 2) теоремы 1.

В характеристике 0 достаточно условия 2) теоремы 1.

Теперь мы можем уточнить результат [1, теорема 10] о точках неопределенности рационального морфизма $g: X \rightarrow Y$.

СледСТВИЕ 5. Предположим, что $K+B$ лог-терминален при некоторой граниче $B$. Тогда образ $g(x)$ замкнутой точки точки $x \in X$ рачионально связен при выполенении предположсений 1), 2) теоремы 1. (Для связности $g(x)$ достаточно нормальности $X$.)

В характеристике 0 достаточно условия 2) теоремы 1.

ДокАЗАТЕльство. Заменим $X$ на разрешение неопределенности рационального морфизма $g$ :

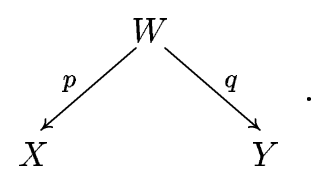

Тогда $g(x)=q\left(p^{-1} x\right)$ и $p^{-1} x$ рационально связно по следствию 3.

Как и следствие 2, выводим

СлЕдСТВИЕ 6. В характеристике 0 рациональная связность в следствиях 3-5 верна при $\operatorname{dim} X \leqslant 3$ без дополнительных предположсений 1), 2) теоремы 1.

Чтобы обобщить следствие 5 до отображения рационально связных множеств (см. следствие 7), надо уметь поднимать рациональные кривые до подобных кратных сечений. Более прямолинеен (ср. с замечанием 6)

Вопрос 1 (ср. [4, вопрос (2)]). Пусть $f: X \rightarrow S$ - стягивание, удовлетворяющее условиям 1)-4) гипотезы. Всякая ли кривая на $C \subseteq S$ имеет сечение над общей точкой?

В случае утвердительного ответа [4, теорема 2.13] дает следующее усиление.

УТВЕРЖДЕНИЕ А (аналог китайской теоремы об остатках). Точнее, существует такое сечение через конечное число точек пространства $X$ в разных гладких слояx $f$ над гладкими точками $C$.

ПримеР 2. По теореме Тзена вопрос 1 имеет утвердительный ответ для $\mathbb{P}^{m}$-расслоений и моноидальных преобразований в неособых центрах.

Кое-где вьше мы строили подобные сечения для рациональны кривых по замечанию 1.

Чтобы построить рациональные кратные сечения в стиле замечания 1 , мы проводим редукцию к относительньм многообразиям $Ф$ ано над кривой $C=P$. Этот подход плохо работает и в случае сечений. Желательна и довольно правдоподобна 
ФоРМУЛА ПРИСОЕДИНЕНИЯ. Если подпространство Y имеет лог-отличие 0, mo

$$
\left.(K+B)\right|_{Y}=K_{Y}+B_{Y}
$$

для некоторого әффективного дивизора $B_{Y}$, при этом $K_{Y}+B_{Y}$ лог-каноничен там, где лог-каноничен $K+B$.

ПРЕДЛОЖЕНИЕ 1. При выполнении формулы присоединения, предположений 1), 2) теоремы 1, и лишь 2) в характеристике 0, утвердительность ответа на вопрос 1 в размерности $\leqslant n=\operatorname{dim} X$ достаточно проверить для расслоений Фано размерности $\leqslant n-1$ над (общей точкой) кривой $C=S$.

УТВЕРЖДЕНИЕ В. Вопрос 1 имеет утвердительный ответ в размерности $\leqslant 3$ ( с заменой сечений на чисто несепарабельные кратные сечения в положительной характеристике).

ДокАЗАТЕЛЬСТво. Увеличивая лог-особенность $X$ над $C$, можно добится появления лог-отличия 0 вдоль подпространства $Y / C$, сохранив при этом лог-каноничность и отрицательность $K+B$ над общей точкой $C$. Применяя формулу присоединения (1), сводим задачу к многообразию Фано и даже Кавамата, лог-терминальному над общей точкой $C$. При ограничении получаем стягивание над общей точкой $C$, как в [11] (cp. [7, лемма 3.6]). В частности, оно задает сечение, когда бирационально.

Утверждение В в случае неособых дель Пецю или рационально связных поверхностей справедливо согласно Кольо-Телену [12]. Общий случай дель Пещцо с лог-терминальными особенностями по теореме 2 (приведенную компоненту границы можно немного уменьшить) сводится после минимального раздутия к неособым рационально связных поверхностям, а потому неособьм дель Пеццо или расслоению на коники. Далее, согласно известным рассуждениям база расслоения на коники будет геометрически (приведена) неприводима и рациональна (ср. определение 1 ; см. также замечание 9). Заметим, что в случае бирационального стягивания-сдутия дивизора $E$ на сечение $C$ дивизор $E$ будет иметь сечение как $\mathbb{P}^{1}$-расслоение.

В случае расслоения на коники дважды применяем теорему Тзена.

В случае не лог-терминальности лог-каноноческая особенность и задает сечение [7, теорема 6.9].

(Ниже в следствиях 7-9 $X$ предполагается неприводимым, по существу нормальным; о полунормальном варианте см. в замечании 4.) Из методов доказательства следствия 5 получаем

СлЕДСТВИЕ 7. В предположениях следствия 5 и

1) при утвердительном ответе на вопрос 1 в размерности $\leqslant n-1$, где $n=\operatorname{dim} X$,

для рачионально связного $P \subseteq X$ образ $g(P)$ тожсе рачионально связен.

СледСТВИЕ 8. В предположениях следствия 5 и условии 1) следствия 7 любая модель пространства $X / S$ рационально связна над точкой базы $S$, когда последнее верно для $X / S$. Значит, рачиональная связность слоя не зависит от модели $c$ лог-терминальными особенностями. 
До размерности 4 вопрос 1 можсно опустить по утверждению В (ср. с замечанием 6).

По замечанию 1 рациональная связность относительного многообразия лог-Фано индуцирована кривыми произвольного разрешения. Откуда получем

ЗАмЕчАнИЕ 2. Последнее утверждение о независимости рациональной связности верно для общего слоя относительного многообразия лог-Фано, т.е. при условиях 1)-4) гипотезы, условиях теоремы 1 и предположении о лог-терминальности особенностей. Отсюда вытекает, что все известные рациональные связности в данной ситуации эквивалентны. В частности, тогда согласно [4, теорема 2.1] две общие точки одной неприводимой компоненты общего слоя можно соединить одной рациональной кривой, а не только их цепочкой. Имеется аналог данного утверждения и для слабого относительного многообразия $Ф$ ано в стиле теоремы 2.

В характеристике 0 достаточно условия 2) теоремы 1.

По существу, указанные результаты относятся к случаю относительного (слабого) многообразия лог-Фано, т.е. $S=$ pt. Однако они могут быть не верны для специальных слоев. Простейший пример - вырожденные слои расслоения на коники. Более интересен пример раздутия трехмерной точки с эллиптическим конусом $E$ в качестве исключительного дивизора. Такое раздутие получется раздутием неособой точки, затем неособой кубики на раздутом дивизоре с последующим сдутием в точку первого. Полученная особенность лог-терминальна. Может быть, имеется подобный пример и с терминальными особенностями. Тем не менее две точки на поверхности $E$ можно соединить двумя рациональньми кривьми $C_{i}$ ограниченной степени: $\left(K_{i} \cdot C_{i}\right)>-1$ (cp. с замечанием 8$)$. Подобньй пример до сдутия с гранищей, сосредоточенной в двух посроенных исключительных дивизорах, показьвает, что неприводимая компонента, отвечающая $E$, не рационально связна, а таков лишь слой в целом.

Можно ожидать также, что рационально связна во всех смыслах неприводимая компонента слоя, которая входит в лог-особенности $K+B$, и $K+B$ лог-терминален. По формуле присоединения это эквивалентно предыдущему (ср. доказательство следствия 3 и замечание 4).

По утверждению В имеем

СлЕдСТВИЕ 9. В характеристике 0 следствия 7, 8 верны при $\operatorname{dim} X \leqslant 3$ без дополнительных предполохсений 1), 2) теоремы 1 и не зависят от ответа на вопрос 1.

ОПРЕДЕЛЕНИЕ-ПРИМЕРЫ. Алгебраическое пространство будем назьвать сильно унилинейчатым, если унилинейчат его образ при любом рациональном морфизме.

В размерности 1 это равносильно рациональности. В характеристике 0 и размерности $\leqslant 2$ верно то же самое.

Однако в размерности 3 лиш в характеристике 0 сильная унилинейчатость равносильна тому, что все образы рациональных морфизмов имеют кодаирову размерность $-\infty$ (в последнем случае можно говорить об абсолютной кодаировой размерности $-\infty$ ) [13, теорема 6.1]. Предположительно то же самое верно и в высших размерностях. 
В положительной характеристике уже унилинейчатость неравносильна отрицательности кодаировой размерности в размерности $\geqslant 2$. Например, существуют унилинейчатые, даже унирациональные К3 поверхности [14].

Отметим, что всякий рациональной морфизм сохраняет унилинейчатость и сильную унилинейчатость (ср. следствие 7).

Вопрос 2 (ср. [4, вопрос (1)]). Верно ли, что алгебраическоепространство $X$ рационально связано при лог-терминальности его особенностей или $K+B$, если и только если оно сильно унилинейчато? Относительная версия может быть верна лишь для общих слоев, отчасти, в силу бирациональности самого утверждения (ср. с замечаниями 2 и 4).

Равносильно, если $X$ не рационально связно, то имеется ли рационально собственный и доминирующий $X \rightarrow Y$, где $Y$ не унилинейчато? В частности, $\operatorname{dim} Y \geqslant 1$ и в характеристике 0 кодаирова размерность $Y$ неотрицательна (см. утверждение 1) предложения 2). Последний морфизм задается отношением рациональной связности [4], [5].

ПРЕДЛОЖЕНИЕ 2. В характеристике 0 утвердительный ответ на вопрос 2 в размерности $\leqslant n$ вытекает из утвердительного ответа на вопрос 1 и предположения 2) теоремы 1 , если помимо того в размерности $\leqslant n$ выполнено утверждение:

1) унилинейчатость равносильна отрицательности кодаировой размерносmu.

ДоКАЗАТЕЛЬСТво. Из рациональной связности пространства вытекает унилинейчатость его образов для регулярных морфизмов. По следствию 8 это верно и для рациональных морфизмов.

Предположим обратное, что $X$ сильно унилинейчато. Тогда по Хиронаке, СГЛМ и следствию 8 можно считать, что пространство $X$ имеет лишь лог-терминальные и даже терминальные особенности. Более того, (лог-) терминальные перестройки в СГЛМ сохраняют рациональную связность. С другой стороны, по предположению 1) $X$ имеет отрицательную кодаирову размерность и по предположению 2) теоремы 1 (после перестроек) расслаивается на многообразия Фано стягиванием $X \rightarrow Y \operatorname{cim} Y<\operatorname{dim} X$.

По предположению $Y$ тоже сильно унилинейчато. Значит, по индукции рационально связно. Отсюда по теореме 1 при утвердительном ответе на вопрос $1 X$ тоже рационально связно. Здесь негласно добавлена гипотеза о полуобильности к СГЛМ.

СлЕдствиЕ 10 (ср. [4, предложение 2.12]). В характеристике 0 при лог-терминальности $X$ или $K+B u \operatorname{dim} X \leqslant 3$ рачиональная связность $X$ әквивалентна сильной унилинейчатости.

ЗАмЕчАниЕ 3. Катсура проверил утверждение следствия 10 в положительной характеристике и размерности 2 .

ЗАмЕчАниЕ 4. В относительном случае утверждение 1) предложения 2 следует заменить на подходящую лог-версию, поскольку при индукции возникнут полунормальные многообразия с кодаировой размерностью в лог-смысле. По-существу это - полунормальный случай (ср. замечание 2). При этом 1) неверно, если унилинейчатость понимать классически. 
Следствия 7-9 тоже можно обобщить на полунормальный случай в подходяшей логкатегории (регулярность рациональньх морфизмов в обших точках ненормальности).

СлЕДСТВИЕ 11. В предложении 1, предполагая выполненным утверждение 1) предложения 2 и формулу присоединения (1), мохно ограничиться случаем первой специи: $\rho(X / C)=1$ и считать, что $X$ имеет лишь терминальные особенности в обшем слое над $C$.

ДокАЗАТЕЛЬСтво. Совместим доказательства утверждения В и предложения 2. В случае лог-терминальных особенностей рациональная связность слоев $X / C$, а потому и сильная унилинейчатость вытекает из следствии 1 и 8.

В не лог-терминальном случае надо использовать формулу присоединения на логособенности и тот факт, что стягивание $X \rightarrow C$ индуцирует стягивание на минимальном центре лог-особенностей (ср. [7, лемма 3.6]).

ЗАМЕчАНИЕ 5. В следствии 11 можно опустить формулу присоединения и предположение 1) предложения 2, когда $\operatorname{dim} X / S \leqslant 2$, воспользовавшись для этого теоремой 1 и [12] (cp. [4, предложение 2.12]).

ЗАмЕчАниЕ 6 . Согласно следствию 11 возможные контрпримеры к утвердительному ответу на вопросы 1 и 2 следует искать для четырехмерных расслоений $Ф$ ано первой специи $X / C$ над кривой $C=\mathbb{P}^{1}$ с терминальными особенностями в общем слое $/ C$. Они могут рассматриваться как трехмерное многообразие Фано над полем $k(C)=k(x)$ рациональных функций одной переменной $x$. Всякое ли такое многообраие Фано имеет (геометрическую) точку над $k(x)$ ?

Однако, может быть, имеется рациональноекратное сечение: точка над $k\left(x^{\prime}\right) \supset k(x)$.

Если такой контрпример существует в размерности $n$ для вопроса 1 (значит, и в размерности $\geqslant n$; как мы уже знаем, $n \geqslant 4)$, то конус над ним с подходящим раздутием вершины приведет к контрпримеру для вопроса 2 в размерности $n+1$ (значит, и в размерности $\geqslant n+1$; ср. пример-замечание и пример 1). Откуда можно усмотреть эквивалентность вопросов 1 и 2 . Отсюда также следовало бы нарушение независимости рациональной связности в размерности $\geqslant n+1$ уже при Кавамата лог-терминальых особенностях, а возможно, и при терминальных (ср. следствие 8).

ЗАмЕчАниЕ 7. Задача о сечении (вопрос 1) и ее усиление (утверждение А) (в случае базы кривая $C=S$, как в предложении 1 ) напоминают соответственно следствие принципа Хассе и китайскую теорему об остатках для (лог-) многообразия Фано $X$ над функциональным полем $K=k(C)$, где $k$ - основное поле, предполагаемое пока алгебраически замкнутым.

В аналогичном случае для арифметического поля $K$ (конечном расширении $\mathbb{Q}$ ) прин-

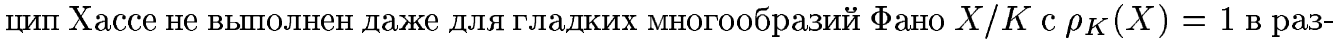
мерности $\geqslant 2[15]$.

ЗАмечАниЕ 8. Для метода Фано важна неприводимость и ограниченность степени (например, относительно поляризации $-K$ или ее лог-версии $-K-B$ ) рациональной цепочки $C$, соединяющей две общие точки в $X$ (или соответственно общую с некоторой). Общие методы нетеровой индукции позволяют утверждать лишь ограниченность 
числа $d$ (соответственно $d^{\prime}$ ) неприводимых компонент $C$, а именно $d \leqslant 2 n-1$ (соответственно $\left.d^{\prime} \leqslant n\right)$, где $n=\operatorname{dim} X$, при неприводимости $X$, и суммою таковых оценок по неприводимым компонентам $X$ (или по неприводимьм компонентам слоя $X / S$ ) в полунормальном случае. Более того, по замечанию 2 следует ожидать, что общие две и более точек можно соединить одной рациональной кривой $C$. Однако ее степень $d=-(K . C)$ неограничена, если мы потребуем лишь лог-терминальность особенностей, что видно уже на рациональных конусах в размерности 2. Конечно, это связано с неограниченностью семейства таких многообразий Фано. Согласно гипотезе Алексеева-Борисова при $\varepsilon$-лог-терминальности с $\varepsilon>0$ семейство соответствующих многообразий лог-Фано размерности $n$ ограничено. Откуда бы следовала ограниченность степеней для границы $d(\varepsilon, n)$ в зависимости от $\varepsilon$ и $n$ ? Какова она? Возможно $d(\varepsilon, n) \leqslant c / \varepsilon$, где $c$ зависит лишь от $n$. В общем случае также возможно, что $d$ ограничено при фиксированном индексе и гранища линейна по индексу.

Однако более привлекательно соединять точки ограниченными цепочками, т.е. общая длина $d=-(K . C)$ или $d=-(K+B . C)$ в лог-версии $\left(\right.$ соответственно $\left.d^{\prime}\right)$ цепочки $C$ ограничена. Точнее, ограничено число $m$ (соответственно $m^{\prime}$ ) компонент цепочки $C$ и степень ее компонент $d$ (соответственно $d^{\prime}=d$ ) для общих двух точек (cp. [3, определение 5.1]). (В таком варианте данный вопрос обобщается на слабые многообразия логФано теоремы 2.) Например, $m \leqslant 2 n-1$ (соответственно $m^{\prime} \leqslant n$ ) и $d \leqslant 2 n$ ? (Заметим, что общая длина тогда не превосходит $4 n^{2}-2 n$; соответственно $2 n^{2}$, что отвечает неособому случаю.) Случай поверхностей хорошо известен: $m \leqslant 3\left(m^{\prime} \leqslant 2\right)$ и $d \leqslant 3$. Для трехмерий $m \leqslant 5\left(m^{\prime} \leqslant 3\right)$ и $d$ ограничено константой. Какой? Автору пока не известно. Известно лишь, что почти всегда (по-видимому с точностью до ограниченного семейства) $d \leqslant 6$. Последнее далеко не тривиально и заслуживает специального рассмотрения, вероятно в одном из последующих писем. Доказательство использует помимо СГЛМ технику дополнений и формулу присоединений для расслоений Фано.

В неособом случае ограниченность $d \leqslant n+1$ известна по деформационным методам Мори [16]. Это влечет и существование ограниченньг неприводимых кривых в неособом случае [5].

ЗАмЕчаниЕ 9. Большая часть утверждений верна над произвольным (не алгебраически замкнутьм) основным полем $k$, если под цепочкою рациональных кривых понимать кривую $C \subseteq X$, определенную над $k$, для которой ее алгебраическое замькание $C_{\bar{k}}$ таково.

ЗАмЕчАнИЕ 10 . В размерности $\leqslant 2$ можно опускать предположение 1) теоремы 1 ; то же самое в размерности 3 и характеристике $\neq 2,3$ и 5 по Абьянкару [17].

\section{СПИСОК ЦИТИРОВАННОЙ ЛИТЕРАТУРЫ}

[1] Shokurov V. V. Letters of a bi-rationalist. I. A projectivity criterion // Cont. Math. 1997. V. 207. P. 143-152.

[2] Манин Ю. И. Кубические формы. М.: Наука, 1972.

[3] Campana F. On twistor spaces of the class C // J. Diff. Geometry. 1991. V. 33. P. 541-549.

[4] Kollár J., Miyaoka Y., Mori S. Rationally connected varieties // J. Algebraic Geometry. 1992. V. 1. P. 429-448. 
[5] Campana F. Connexité rationnelle des variétés de Fano // Ann. Sci. Éc. Norm. Sup. 1992. V. 25. P. 539-545.

[6] Campana F. Un théorèm de finitude pour les variétés de Fano suffisamment uniréglées // Preprint, 1996.

[7] Шокуров В. В. Трехмерные логперестройки // Изв. АН СССР. Сер. матем. 1992. Т. 56. C. $105-203$.

[8] Shokurov V. V. Anticanonical boundedness for curves. Appendix to V. V. Nikulin "Hyperbolic reflection group methods and algebraic varieties" // Higher Dimensional Complex Varieties. Proceedings of the International Conference held in Trento, Italy (June 15-24), 1994. P. 321-328.

[9] Shokurov V. V. 3-fold log models // J. Math. Sciences. 1996. V. 81. P. 2667-2699.

[10] Kawamata Y., Matsuda K., Matsuki K. Introduction to the minimal model problem // Adv. Stud. Pure Math. 1987. V. 10. P. 283-360.

[11] Kollár J. et al. Flips and abundance for algebraic threefolds. Astérique. V. 211, 1992.

[12] Colliot-Theèléne J.-L. Arithmétique des variétés rationelles et problèmes birationnelles // Proc. Int. Conf. Math. 1986. P. 641-653.

[13] Kollár J. Higher direct images of dualizing sheaves // Ann. of Math. 1986. V. 123. P. 11-42.

[14] Katsura T. Theorem of Luroth and related topics // Algebraic Geometric Seminar. Singapore: World Scientific, 1987. P. 41-52.

[15] Birch B. J., Swinnerton-Dyer H.P. F. The Hasse problem for rational surfaces // J. Reine Angew. Math. 1975. V. 274. P. 164-174.

[16] Mori S. Projective manifolds with ample tangent bundles // Ann. Math. 1979. V. 110. P. 593-606.

[17] Абьлнкар Ш.Ш. On the problem of resolution of singularities // Тр. Международного Конгресса математиков. М., 1966. С. 469-481. 